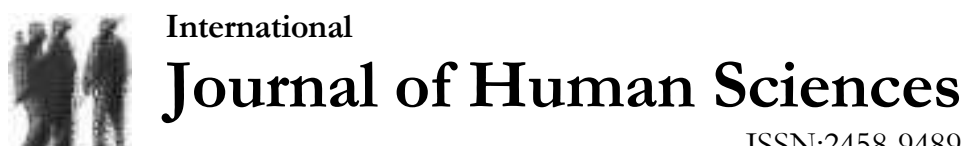 \\ ISSN:2458-9489
}

Volume 17 Issue 1 Year: 2020

\section{Effect of process recording and self-compassion on self- focus: A pre-post interventional pilot study}

\author{
Yusuke Kurebayashi ${ }^{1}$ \\ Yusuke Harada ${ }^{2}$
}

\begin{abstract}
Research Problem/aim: This study examined whether process recording increases self-focus, and whether this effect is moderated by self-compassion.

Methods: Participants included 31 undergraduates in Tokyo. A pre-post-test design was used. Participants completed measures including self-focus and self-compassion before and after process recording. Participants were divided into high and low self-compassion groups. Findings: Overall, the mean rumination and reflection scores were 42.0 and 37.9, respectively, at pre-intervention. The low self-compassion group $(n=14)$ showed no significant changes in either subscale. The high self-compassion group $(n=17)$ showed a significant reduction in rumination.
\end{abstract}

Conclusions: Overall, process recording does not influence self-focus, but does reduce rumination in those with high self-compassion. Enhancing self-compassion is needed before using process recording to prevent excessive rumination.

Keywords: Rumination; self-compassion; reflection sheet; nursing education; mindfulness.

\section{Introduction}

With the development of healthcare technology, nurses have needed to continually improve their nursing performance. Research has shown that scientific thinking (Tatsumi, Nakama, Tada, \& Koyama, 2008), years of experience in nursing (Tamura, Tanaka, Tanaka, Horiuchi, 2010), social skills (Masuhara et al., 2007), self-education (Nakajima et al., 2004), autonomy (Tsuji et al., 2007), and occupational stress (Kurebayashi, Harada, \& Inoue, 2016) as well as other factors are relevant factors associated with improvements in nursing competency. Self-reflection is another important factor that has received attention. Sugiyama and Asakura (2017), using the modified grounded theory approach, analyzed interviews with 14 nurses with over 8 years of clinical nursing experience, and found that reviewing judgments was an important part of nurses' process of autonomous clinical judgment (i.e., judgments made by nurses without the control or support of other medical staff). Eng and Pai (2015) investigated nursing undergraduates in Taiwan and found that higher self-reflection was associated with better nursing competency. Furthermore, Pai (2015) conducted a self-reflection program on nursing students before their clinical practicums, and

\footnotetext{
${ }^{1}$ RN, Ph.D. Lecture, Niigata University of Health and Welfare, Faculty of Nursing, yusukekure@yahoo.co.jp

2 OTR, Ph.D. Assistant Professor, Kyorin University, Faculty of Health Sciences, Department of Occupational Therapy, y-harada@ks.kyorin-u.ac.jp
} 

interventional pilot study. Journal of Human Sciences, 17(1), 131-141. doi:10.14687/jhs.v17i1.5753

reported that better self-reflection was associated with higher nursing competency at 6 months after the clinical practicum. The bottom line seems to be that self-reflection is associated with better nursing competency.

In psychology, focusing on the self is referred to as "self-focus." Specifically, according to Sakamoto (1997), self-focus is "the state of focusing on oneself and one's character traits predisposing one to this state." Self-focus can be divided into two domains: "self-reflection," an adaptive aspect motivated by intellectual curiosity, and "rumination," a maladaptive aspect motivated by threat and loss. There is a robust, positive association between rumination and depression (Hasegawa et al., 2018). Furthermore, higher nursing skills in psychiatric nurses has been associated with higher reflection and lower rumination (Kurebayashi, 2019). Therefore, nursing educators should focus on preventing excessive rumination and enhancing self-reflection to improve nursing competencies among nursing students during their education.

One long-used technique for facilitating self-reflection on nursing care employed in nursing education is called "process recording." Among the functions of process recording and its associated supervision is the development of self-awareness (Bodley, 1992). Process recording involves using a reflection sheet to record interactions between nurses and patients, including patients' speech and behavior, nurses' own feelings and thoughts in response to such speech and behavior, and nurses' overall reactions to patients. Nursing students attempt to reflect and understand the following points via process recording: patients' feelings and thoughts; their own tendencies; and whether they exhibit behavior and speech in concordance with their own feelings (Miyamoto, 2003; Townsend, 2011). In summary, process recording requires individuals to focus on themselves.

However, by promoting self-focus, process recording offers the opportunity to increase rumination as well as self-reflection. In performing process recording for clinical practice, some students tend to focus excessively on their own lack of skill or weaknesses. Bodley ${ }^{13}$ pointed out that process recording can present problems to students, depending on their experience and degree of hyper-sensitivity. However, Shattel, Hogan, and Hernandez (2006), in their review, pointed out that there are no studies examining the effect of process recording. This means that it is still unclear whether process recording leads to adaptive or maladaptive self-focus. It might also be necessary to devise ways of preventing excessive rumination during process recording.

Recently, self-compassion has been found to have a protective effect against mental health problems. Self-compassion refers to the process of showing compassion to oneself. It consists of three aspects: self-kindness, which is extending kindness and understanding toward oneself rather than engaging in self-criticism and judgment; common humanity, which is viewing one's experiences as part of the larger human experience rather than as separating and isolating; and mindfulness, which is viewing painful thoughts and feelings with balanced awareness rather than over-identifying with them (Neff, 2003). Ehret, Joormann, and Berking (2015) compared selfcompassion between healthy people and people with major depressive disorder, and found that the latter group had significantly lower self-compassion than did the former. Furthermore, higher selfcompassion has been associated with lower burnout in counseling students (Beaumont, Durkin, Hollins Martin, \& Carson, 2016) and community nurses (Durkin, Beaumont, Hollins Martin, \& Carson, 2016). Together, this research suggests that self-compassion can prevent excessive rumination.

Although process recording has been widely introduced in nursing schools worldwide, the effect of process recording and the moderating effects of self-compassion has not been examined empirically. Nursing education would benefit from understanding the effect of process recording on self-focus, and as well as whether self-compassion moderates this effect. However, these two points remain unclear. Therefore, this study aimed to address (1) whether process recording affects self-focus, and (2) whether self-compassion moderates the effect of process recording on selffocus, using a pre-post-intervention design with Japanese nursing students. 


\section{Methods}

\section{Participants}

Participants were junior and senior nursing undergraduates from Tokyo, Japan. In the nursing education curriculum in Japan, junior and senior students are taught to use process recording, and are supervised by teachers while performing process recording in their clinical practicums. To recruit participants, a researcher announced and asked students to participate at the end of their class. Students that were willing to participate were asked to meet in another room to complete the study.

\section{Study Design}

This was a pretest-post-test interventional design. Participants were instructed to complete the study questionnaire before and after a session of process recording.

\section{Measurements}

Demographics and attitudes toward use of process recording. The demographic characteristics assessed were age and the clinical practicum they were most impressed with. We also assessed their feelings about using process recording (which had three answer options: "process recording is not enjoyable," "I have no problem with process recording," and "process recording is enjoyable") and their perceptions of using process recording ("process recording is not beneficial," "process recording is not particularly beneficial," "process recording is beneficial," and "process recording is very beneficial").

Self-focus. Self-focus was investigated using the Japanese version of the Rumination-Reflection Questionnaire (RRQ), which has good reliability and validity (Takano \& Tanno, 2008). ${ }^{21}$ We used the RRQ because it is used worldwide and can detect both the positive and negative aspect of selffocus (reflection and rumination, respectively), which aligns with our intention to determine whether process recording influences both reflection and rumination.

The RRQ comprises rumination and reflection subscales; in other words, the RRQ can capture both the adaptive and maladaptive aspects of self-focus. The subscales each comprise 12 items, all of which are rated on a 5 -point Likert scale $(1=$ strongly disagree to $5=$ strongly agree $)$. Higher scores indicate a more self-focused character. The subscale scores were used in the analysis as the primary outcomes, and therefore were measured at both time points (i.e. before and after intervention).

Self-compassion. As a moderating factor, self-compassion was measured using the Japanese version of the Self-Compassion Scale (SCS), which is the most used scale worldwide for measuring self-compassion. The SCS comprises six subscales: self-kindness, self-judgment, common humanity, isolation, mindfulness, and over-identification. Self-kindness, common humanity, and mindfulness are considered the components of self-compassion, whereas self-judgment, isolation, and over-identification are opposing (i.e., negative) concepts. In this study, we used only the scores on the self-kindness, common humanity, and mindfulness subscales. All 26 items are rated on a 5point Likert scale $(1=$ almost never to $5=$ almost always). Higher scores on these three scales indicate higher self-compassion. The SCS was administered only before the intervention. The validity and reliability of the SCS Japanese version have been examined (Arimitsu, 2014). The Cronbach's alpha of the whole SCS was .839, and for the subscales, it ranged from .718 to .820 . For the subscales, the coefficients are considered relatively low. However, the Cronbach's alpha for all subscales and the whole scale was more than .7 , which is the general threshold for concern with the Cronbach's alpha. Furthermore, the SCS otherwise has good reliability. Therefore, we used the SCS Japanese version in this study. 

interventional pilot study. Journal of Human Sciences, 17(1), 131-141. doi:10.14687/jhs.v17i1.5753

\section{Intervention}

Participants were asked to participate in writing process recording only one time as the intervention in this study. They were given directions to reflect on a scene during their clinical practicum that they found most impressive about interaction with a patient. Specifically, they were asked to note the following points on the process recording sheet, without supervision: time, place, and environment of the scene; summary of all interactions with the patients; the reason that they chose the scene; the speech and behavior of the patients; their own thoughts, feelings, speech, and behavior; and a reflection on and analysis of the scene or interactions therein. To guarantee that the quality of the intervention was uniform, the same researcher conducted all parts of the intervention. Lectures on how to use and perform process recording and its merits for nursing performance were conducted when students were in their second year. Furthermore, students actually used and practiced process recording in their clinical practicum in the third year of university, meaning that participants in this study had been taught about process recording and had experienced using it before the study. The intervention was conducted when participants were in between clinical practicums, meaning that participants were not participating in a clinical practicum at the time of the study.

\section{Required Term of Study Protocol}

The required term of study protocol (from pre-test to post-test) as participants was approximately 45 minutes.

\section{Study Duration}

The study was conducted from September to November 2017.

\section{Statistical Analysis}

Participants were first divided into two groups (high and low SCS) according to whether their mean score on the positive aspect of the SCS was above or below the cut-off of 8.7. This cutoff was chosen by referring to the Japanese mean score in a large-scale study by Arimitsu (2014). We then compared the means scores of the RRQ at pre-intervention with those at postintervention using paired $t$-tests in the total sample and the high and low SCS groups. Although the sample was relatively small, we used parametric analyses because a recent advocated for their general use, save for in instances where the data show an extreme deviation from the normal distribution (Pranee, 2010). All statistical analyses were performed using SPSS Statistics 23.0 for Windows (IBM Corp., Armonk, NY, USA). The significance level was set at 5\%.

\section{Ethical Consideration}

All participants were informed of the following via explanatory documents given to them before participation: research aim, methods, voluntary nature of participation, the fact that they could leave the study early, the fact that individual data would never be shown, and the lack of any consequences for academic evaluations if they chose to refuse participation. Participants were asked to answer the questionnaire if they wanted to participate in the study. Before we began data collection, the study protocol was approved by the ethical committee of the university at which the corresponding authors worked.

\section{Results}

\section{Descriptive Statistics and Internal Consistency at Pre-intervention}

Thirty-one undergraduates participated in this study. As shown in Table 1, their mean (SD) age was 21.7 (0.6) years old. As for the clinical practicum that students found most impressive, the largest proportion of students reported the practicum dealing with nursing care for chronic illness in adults $(n=9,29.0 \%)$. Most participants reported that "I have no problem with process 
Kurebayashi, Y., \& Harada, Y. (2020). Effect of process recording and self-compassion on self-focus: A pre-post interventional pilot study. Journal of Human Sciences, 17(1), 131-141. doi:10.14687/jhs.v17i1.5753

recording" ( $n=22,71.0 \%)$, and "process recording is beneficial" ( $n=19,61.3 \%)$. The mean scores on the RRQ and SCS at pre-intervention are shown in Table 1. At pre-intervention, the Cronbach's $\mathrm{a}$ of the rumination and reflection subscales of the RRQ along with the positive SCS ranged from .704 to .903 . This indicated relatively good reliability.

Table 1. Descriptive statistics at pretest $(n=31)$

\begin{tabular}{|c|c|c|c|c|}
\hline & $\begin{array}{r}\text { mean } \\
\mathrm{n}\end{array}$ & & $\begin{array}{l}\text { SD } \\
\%\end{array}$ & \\
\hline Age & 21.7 & \pm & 0.6 & \\
\hline \multicolumn{5}{|l|}{ Rumination-Reflection Questionnaire } \\
\hline pre RRQ Rumination subscale $(\alpha=.704)$ & 42.0 & \pm & 5.9 & \\
\hline pre RRQ Reflection subscale $\quad(\alpha=.902)$ & 37.9 & \pm & 9.4 & \\
\hline \multicolumn{5}{|l|}{ Self-compassion Scale } \\
\hline SCS positive $(\alpha=.903)$ & 9.0 & \pm & 2.5 & \\
\hline pre SCS self-kindness & 3.1 & \pm & 0.9 & \\
\hline pre SCS common humanity & 2.9 & \pm & 1.0 & \\
\hline pre SCS mindfulness & 3.0 & \pm & 1.0 & \\
\hline SCS negative $(\alpha=.891)$ & 10.4 & \pm & 2.2 & \\
\hline pre SCS self-judgment & 3.5 & \pm & 0.9 & \\
\hline pre SCS isolation & 3.3 & \pm & 1.0 & \\
\hline pre SCS over-identification & 3.6 & \pm & 0.8 & \\
\hline \multicolumn{5}{|l|}{ Clinical practicums most impressed with } \\
\hline Fundamental nursing & 3 & & 9.7 & ) \\
\hline Critical care nursing & 7 & & 22.6 & ) \\
\hline Chronical nursing & 9 & & 29.0 & ) \\
\hline Pediatric nursing & 0 & & 0.0 & ) \\
\hline Maternity nursing & 4 & & 12.9 & ) \\
\hline Psychiatric nursing & 6 & & 19.4 & ) \\
\hline Home care nursing & 1 & & 3.2 & ) \\
\hline Geriatric nursing & 1 & & 3.2 & ) \\
\hline \multicolumn{5}{|l|}{ Feelings about process recording } \\
\hline Process recording is not enjoyable & 8 & & 25.8 & ) \\
\hline Have no problem with process recording & 22 & & 71.0 & ) \\
\hline Process recording is enjoyable & 1 & & 3.2 & ) \\
\hline \multicolumn{5}{|l|}{ Perception of using process recording } \\
\hline Process recording is not beneficial & 1 & & 3.2 & ) \\
\hline Process recording is not particularly beneficial & 8 & & 25.8 & ) \\
\hline Process recording is beneficial & 19 & & 61.3 & ) \\
\hline Process recording is very beneficial & 3 & & 9.7 & ) \\
\hline
\end{tabular}

\section{Changes in RRQ Before and After Intervention}

As shown in Table 2, neither the rumination subscale nor the reflection subscale showed changes at post-intervention in the total sample $(n=31 ; p=.295$ and .428 , respectively). The results of the sub-group analysis were shown in Table 2, Figure 1, and Figure 2. The mean scores on the rumination subscale did show a significant reduction in the high SCS group $(p=.029)$, but not in the low SCS group ( $p=.170$; Table 2, Figure 1). 
Kurebayashi, Y., \& Harada, Y. (2020). Effect of process recording and self-compassion on self-focus: A pre-post interventional pilot study. Journal of Human Sciences, 17(1), 131-141. doi:10.14687/jhs.v17i1.5753

Table 2. Rumination and reflection scores before and after process recording intervention $(\mathrm{n}=31)$

\begin{tabular}{|c|c|c|c|c|c|c|c|c|c|c|c|c|c|c|c|}
\hline \multirow[b]{3}{*}{ Total $(\mathrm{n}=31)$} & \multicolumn{8}{|c|}{ Rumination } & \multicolumn{7}{|c|}{ Reflection } \\
\hline & \multicolumn{3}{|c|}{ pre } & \multicolumn{3}{|c|}{ post } & \multirow{2}{*}{$\begin{array}{c}p \\
.295\end{array}$} & \multicolumn{4}{|c|}{ pre } & \multicolumn{3}{|c|}{ post } & \multirow{2}{*}{$\frac{p}{.428}$} \\
\hline & 42.0 & \pm & 5.9 & 41.4 & \pm & 7.1 & & & 37.9 & \pm & 9.4 & 37.4 & \pm & 10.6 & \\
\hline \multicolumn{16}{|l|}{ SCS Positive } \\
\hline Low $(n=14)$ & 42.6 & \pm & 7.2 & 43.4 & \pm & 6.9 & .302 & & 34.8 & \pm & 9.6 & 35.0 & \pm & 11.5 & .817 \\
\hline $\operatorname{High}(\mathrm{n}=17)$ & 41.5 & \pm & 4.9 & 39.6 & \pm & 6.9 & .029 & $*$ & 40.4 & \pm & 8.8 & 39.4 & \pm & 9.8 & .170 \\
\hline
\end{tabular}

paired t-test

* $\mathrm{p}<.05$

Figure 1. Change in rumination scores in both self-compassion groups $(n=31)$

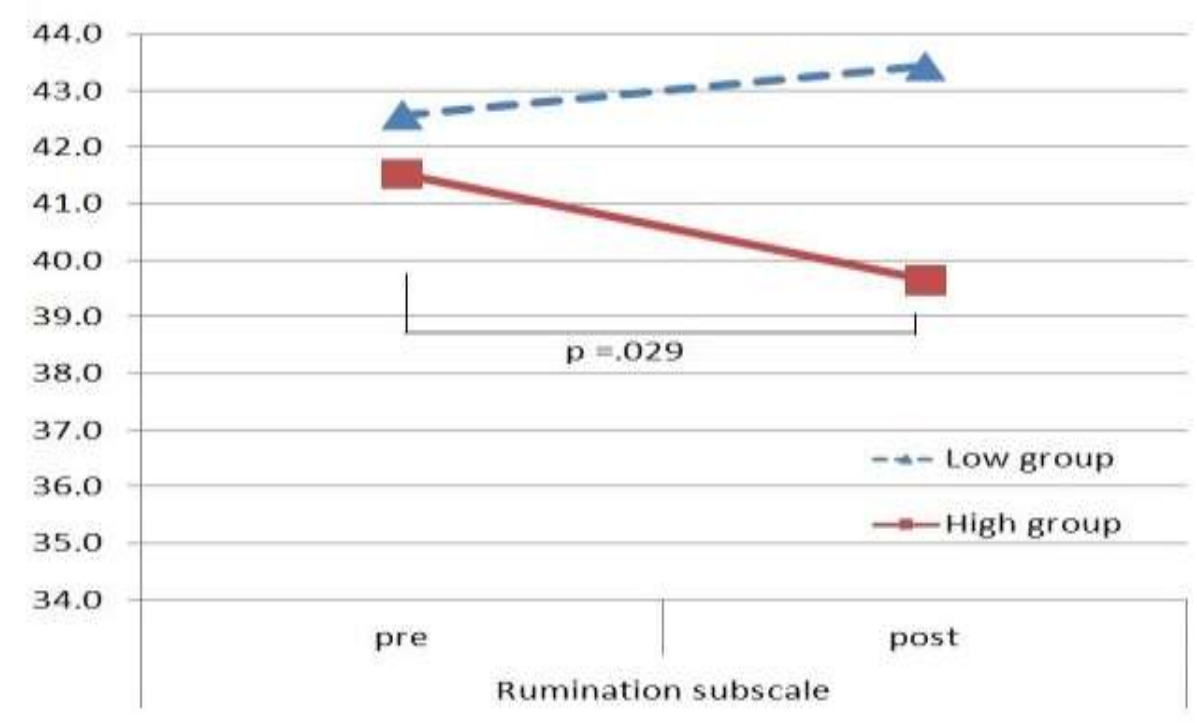

Figure 2. Changes in reflection scores in both self-compassion groups $(n=31)$

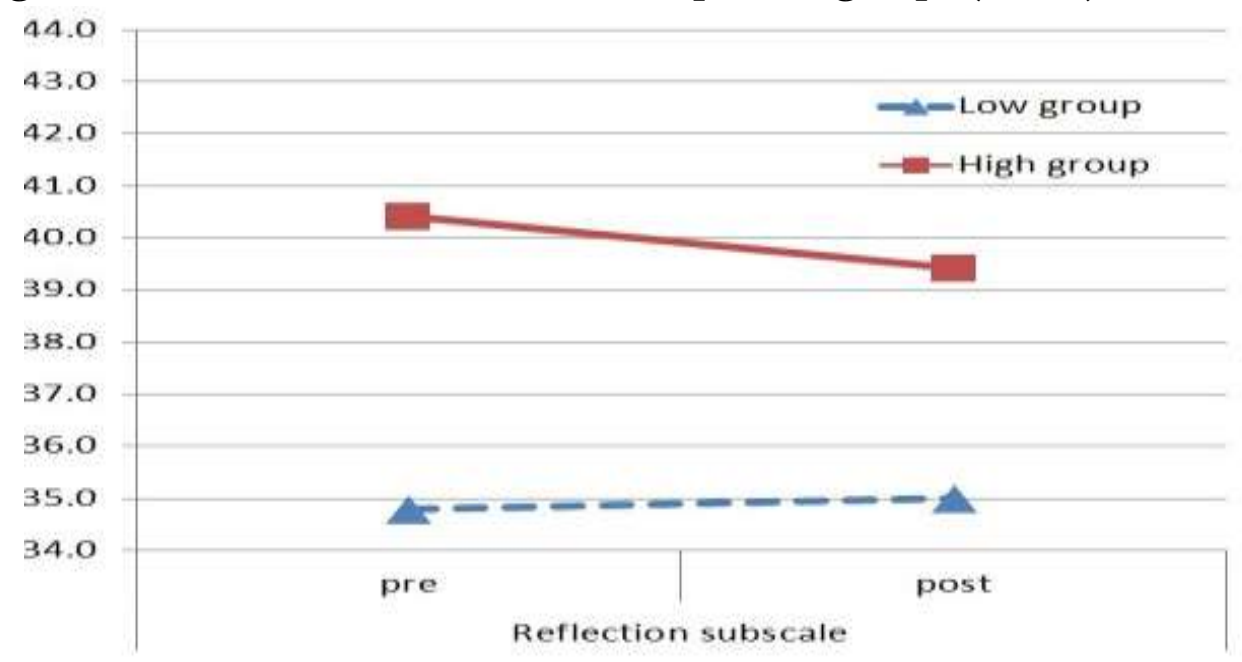




\section{Discussion}

This study is the first to examine the effect of process recording on self-focus and whether self-compassion moderates this effect using an experimental design. There were two main findings. First, the mean score of self-focus for the total sample did not change significantly after the process recording. Second, participants with high self-compassion showed a significant reduction in rumination after process recording.

Several studies have employed mirrors and the sentence completion test as interventions to increase self-focus (Sakamoto, 2010). However, surprisingly, process recording-which has nursing students review their feelings about patients, as well as their own speech and behavior-did not lead to an increase in either rumination or reflection. In other words, process recording does not seem to influence either the maladaptive or adaptive aspects of self-focus.

However, students who have the tendency of to show high self-compassion experienced significantly decreased rumination after the process recording. Considering this study's design was pre-post interventional study and the required term was approximately 45 minutes, the changes may be emerged by process recording as intervention.

Svendsen and colleagues (2016), in a sample of university students, found that higher selfcompassion was associated with lower rumination, leading them to suggest that self-compassion can lead to adaptive emotional responses. Our result supports their findings.

Besides enhancing self-awareness, it is essential to prevent excessive rumination as well. Dundas and colleagues (2017), in a sample of undergraduates, found that those who are high in self-compassion showed lower self-condemnation. Trompetter, de Kleine, and Bohlmeijer (2017) examined 349 members of the general population $\left(M_{\text {age }}=32.88, S D=12.99\right.$ years $)$ in an online survey, and found that higher self-compassion reduced the association between negative affect and psychopathology. Accordingly, self-compassion might attenuate rumination.

Process recording helps boost self-awareness and expression of one's own feelings, which are crucial to improving nursing skills. Breines and Chen (2012) instructed undergraduates to write a response to the following prompt in order to induce self-compassion: "Imagine that you are talking to yourself about your weakness from a compassionate and understanding perspective. What would you say?" They found that the number of expressions relating to their belief about their own weakness was greater in the self-compassion condition than in the no intervention condition. SSenyuva and fellow researchers (2014) reported that higher self-compassion was associated with better self-awareness in nursing students. Furthermore, Krieger and associates (2013) reported that higher self-compassion reduced avoidance. Therefore, higher self-compassion seems to facilitate greater self-awareness.

The reason that rumination was reduced among students with higher self-compassion despite the fact that all participants of process recording must face their own weaknesses and strengths - might be the mediating effect of self-awareness. Several studies have shown that higher self-awareness is associated with lower rumination (DaSilveira, DeSouza, \& Gomes, 2015; Sutton, 2016). Harrington and Loffredo (2010) reported that self-acceptance has a positive association with self-awareness, and a negative association with rumination. Furthermore, our previous qualitative study (2010), which compared categories of reactions to written process recording between two groups of students categorized as having high or low self-compassion, showed that low selfcompassionate students had tendency of "Focusing on one's weakness or limitation," while high self-compassionate students had tendencies of "Recalling warm interaction with patients" and "Gaining new perspectives of interpreting an interaction with patients." Even if the situation might be uncomfortable before process recording, written process record led that high self-compassionate students could objectively analysis the situation selected through process recording. Therefore, only high self-compassionate student might lead to reduce their rumination. Taken together, a potential reason that rumination declines after process recording only in students with high self-compassion might be that self-compassion facilitates self-awareness, which in turn attenuates rumination. 

interventional pilot study. Journal of Human Sciences, 17(1), 131-141. doi:10.14687/jhs.v17i1.5753

\section{Limitations}

This study has several limitations. First, the sample was small. Future studies should use a larger sample, which might help in detecting significant differences. Second, we lacked a control group, so we could not detect practice effects on the RRQ scores. Because practice effects might have influenced the results, future studies should compare a control group with an intervention group. Third, the intervention only involved instructing participants on how to perform process recording, and did not include supervision. In actual nursing education, effective process recording require both the student's personal review of their interaction with patients and adequate supervision. ${ }^{16}$ The results of interventions including both might differ from our results. Fourth, the description content of the process recording was not analyzed. Possibly, self-focus is influenced by certain description content. Future studies should analyze how self-focus is affected by description content in process recording. Fifth, the cut-off point for defining the SCS groups was set according to data obtained from Japanese people. Because the SCS average score would differ by country, our results cannot be generalized to nursing students of other countries. Sixth, the actual effect of process recording in Japan might differ from that in another country. In general, the aims of process recording are to encourage (1) understanding and reflection on oneself as a healthcare provider, (2) understanding of the needs of patients and what patients wish to express, and (3) reflection on the care that students provide. In Japanese facilities, process recording has a sole main aim: that is, to encourage understanding and reflection on oneself as a healthcare provider (Mikamoto, 2003; Townsend, 2011); possibly, this main aim might differ across countries, which could in turn influence the results. This study must be replicated in other countries to generalize the result. Finally, the situations that participants selected for the process recording varied among the participants, and could might have influenced the study results. Nevertheless, we limited the number of possible situations by having participants focus solely on situations during clinical practicums.

\section{Conclusions}

This study found that process recording did not influence self-focus in the total sample. However, it did seem to lead to a reduction in rumination among students with higher selfcompassion. When educating nursing students on the use of process recording, it might be necessary to focus on enhancing self-compassion in order to prevent excessive rumination.

\section{Implications for Nursing Practice}

Before teaching nursing students how to perform process recording, it seems important to enhance their self-compassion in order to prevent excessive rumination, which is associated with worse nursing competency. In general, self-compassion shows a decline over time in adolescence, especially among females (Bluth \& Blanton, 2015; Bluth, Campo, Futch, \& Gaylord, 2017). Furthermore, Murphy and colleagues (2009) reported that the third-year nursing students showed less caring behavior than did first-year nursing students, suggesting that self-compassion and caring behavior decline over time in nursing school. Therefore, nursing education should focus on enhancing self-compassion before teaching process recording (which begins in the second year of nursing school in Japan).

Increasing self-compassion requires consideration of teaching style. Eraydin and Karagözoğlu (2017) compared the effect of three types of nursing curriculum on self-compassion, and found that only the integrated curriculum - which involves teaching basic knowledge, skills, and attitudes related to nursing using small-scale group study - increased self-compassion. They posited that such a curriculum facilitated more effective learning, which in turn enhanced selfcompassion.

Duarte and Pinto-Gouveia (2016) conducted a mindfulness-based intervention with oncology nurses, and found that it increased self-compassion. Compassion-based interventions have also been found to benefit self-compassion. Kirby, Tellegen, and Steindl (2017) conducted a 

interventional pilot study. Journal of Human Sciences, 17(1), 131-141. doi:10.14687/jhs.v17i1.5753

meta-analysis of randomized controlled trials examining whether compassion-based interventions actually improve self-compassion. Although they included interventions targeting compassion toward others and oneself in their meta-analysis, the composite effect of 13 studies $(n=882)$ reached significance $\left(d=.70, p<.001, I^{2}=59.99 \%\right)$. Smeets and colleagues (2014) also reported that an intervention targeting self-compassion was effective in its intended purpose. Their intervention involved teaching knowledge of self-compassion and exercises wherein made personalized phrases based on the three components of self-compassion, listed five reasons they were grateful for themselves, and similar activities.

Referring to these findings, it might be necessary to integrate the three components of selfcompassion into the forms used in process recording or the supervision thereof, as well as having students perform process recording in small groups. For example, questions could include "What would you say to yourself to express compassion during difficult interactions between you and the patient?" "Many students have experience similar difficulties in interacting," and "What is the point of trying to work hard to care for your patients?"

\section{References}

Arimitsu, K. (2014). Development and validation of the Japanese version of the Self-Compassion Scale. Japanese Journal of Phycology, 85, 50-59. https://doi.org/10.4992/ijpsy.85.50

Beaumont, E., Durkin, M., Hollins Martin, C. J., \& Carson, J. (2016). Measuring relationships between self - compassion, compassion fatigue, burnout and well - being in student counsellors and student cognitive behavioural psychotherapists: A quantitative survey. Counselling \& Psychotherapy Research, 16(1), 15-23. https://doi.org/10.1002/capr.12054

Bluth, K., \& Blanton, P. W. (2015). The influence of self-compassion on emotional well-being among early and older adolescent males and females. The Journal of Positive Psychology, 10(3), 219-230. https://doi.org/10.1080/17439760.2014.936967

Bluth, K., Campo, R. A., Futch, W. S., \& Gaylord, S. A. (2017). Age and gender differences in the associations of self-compassion and emotional well-being in a large adolescent sample. Journal of Youth and Adolescence, 46(4), 840-853. https://doi.org/10.1007/s10964-016-0567-2

Bodley, D. E. (1992). Clinical supervision in psychiatric nursing: Using the process record. Nurse Education Today, 12(2), 148-155. https://doi.org/10.1016/0260-6917(92)90042-M

Breines, J. G., \& Chen, S. (2012). Self-compassion increases self-improvement motivation. Personality and Social Psychology Bulletin, 38(9), 1133-1143. https://doi.org/10.1177/0146167212445599

DaSilveira, A., DeSouza, M. L., \& Gomes, W. B. (2015). Self-consciousness concept and assessment in self-report measures. Frontiers in Psychology, 6, 930. https://doi.org/10.3389/fpsyg.2015.00930

Duarte, J., \& Pinto-Gouveia, J. (2016). Effectiveness of a mindfulness-based intervention on oncology nurses' burnout and compassion fatigue symptoms: A non-randomized study. International Journal of Nursing Studies, 64, 98-107. https://doi.org/10.1016/i.ijnurstu.2016.10.002

Dundas, I., Svendsen, J. L., Wiker, A. S., Granli, K. V., \& Schanche, E. (2016). Self-compassion and depressive symptoms in a Norwegian student sample. Nordic Psychology, 68(1), 58-72. https://doi.org/10.1080/19012276.2015.1071203

Durkin, M., Beaumont, E., Hollins Martin, C. J., \& Carson, J. (2016). A pilot study exploring the relationship between self-compassion, self-judgement, self-kindness, compassion, professional quality of life and wellbeing among UK community nurses. Nurse Education Today, 46, 109-114. https://doi.org/10.1016/j.nedt.2016.08.030

Ehret, A. M., Joormann, J., \& Berking, M. (2015). Examining risk and resilience factors for depression: The role of self-criticism and self-compassion. Cognition and Emotion, 29(8), 1496-1504. https://doi.org/10.1080/02699931.2014.992394 
Kurebayashi, Y., \& Harada, Y. (2020). Effect of process recording and self-compassion on self-focus: A pre-post interventional pilot study. Journal of Human Sciences, 17(1), 131-141. doi:10.14687/jhs.v17i1.5753

Eng, D. J., \& Pai, H. C. (2015). Determinants of nursing competence of nursing students in Taiwan: The role of self-reflection and insight. Nurse Education Today, 35(3), 450-455. https://doi.org/10.1016/i.nedt.2014.11.021

Eraydın, S., Karagözoğlu, Ş. (2017). Investigation of self-compassion, self-confidence and submissive behaviors of nursing students studying in different curriculums. Nurse Education Today, 54, 44-50. https://doi.org/10.1016/i.nedt.2017.03.007

Harrington, R., \& Loffredo, D. A. (2010). Insight, rumination, and self-reflection as predictors of well-being. The Journal of Psychology, 145(1), 39-57. https://doi.org/10.1080/00223980.2010.528072

Hasegawa, A., Kunisato, Y., Morimoto, H., Nishimura, H., \& Matsuda, Y. (2018). How do rumination and social problem solving intensify depression? A longitudinal study. Journal of Rational-Emotive \& Cognitive-Behavior Therapy, 36, 28-46. https://doi.org/10.1007/s10942017-0272-4

Kirby, J. N., Tellegen, C. L., \& Steindl, S. R. (2017). A meta-analysis of compassion-based interventions: Current state of knowledge and future directions. Behavior Therapy, 48(6), 778792. https://doi.org/10.1016/i.beth.2017.06.003

Krieger, T., Altenstein, D., Baettig, I., Doerig, N., \& Holtforth, M. G. (2013). Self-compassion in depression: Associations with depressive symptoms, rumination, and avoidance in depressed outpatients. Behavior Therapy, 44(3), 501-513. https://doi.org/10.1016/j.beth.2013.04.004

Kurebayashi, Y. (2019). Comparison of factors predicting nursing skills between general and psychiatric nurses. Perspectives in Psychiatric Care, 55(2), 183-189. https://doi.org/10.1111/ppc.12316

Kurebayashi, Y., \& Harada, Y. (2018). The different reactions to writing process recording between low and high self-compassionate students: A pre-post qualitative analysis. Japanese Journal of Human Sciences of Health-Social Services, 25, 1-8.

Kurebayashi, Y., Harada, Y., \& Inoue, Y. (2016). The relationships between occupational stress and nursing performance in psychiatric nurses. Japanese Journal of Human Sciences of Health-Social Services, 22, 1-8. https://doi.org/10.20681/hwelfare.22.2 1

Masuhara, K., Uchida, H., Masui, E., Tsumoto, Y., Nagata, K., Nagasawa, Y., \& Fukuma, M. (2007). [Development of nursing with nursing performance and social skills]. Bulletin of Shimane University Faculty of Medicine, 30, 51-57, Japanese

Miyamoto, M. (2003). Process recording as methods of caring. Tokyo: Seishinkangosyuppan.

Murphy, F., Jones, S., Edwards, M., James, J., \& Mayer, A. (2009). The impact of nurse education on the caring behaviours of nursing students. Nurse Education Today, 29(2), 254-264. https://doi.org/10.1016/i.nedt.2008.08.016

Nakajima, H., Iwasaki, T., Yanaga, F., Kimura, H., Fujiwara, K., Nishimura, K., ... Chikada, K. (2004). Facilitating clinical competency in middle level nurses. Nihonkangogakkaironnbunsyu: Kangokanri, 34, 207-209.

Neff, K. D. (2003). The development and validation of a scale to measure self-compassion. Self and Identity, 2(3), 223-250. https://doi.org/10.1080/15298860309027

Pai, H. C. (2015). The effect of a self-reflection and insight program on the nursing competence of nursing students: A longitudinal study. Journal of Professional Nursing, 31(5), 424-431. https://doi.org/10.1016/i.profnurs.2015.03.003

Pranee, L. (2010). Research methods in health: foundations for evidence-based practice (1st ed.). South Melbourne: Oxford University Press.

Sakamoto, S. (1997). Self-focus and depression: toward an interface between social and clinical psychology. Tokyo: University of Tokyo Press.

Şenyuva, E., Kaya, H., Işik, B., \& Bodur, G. (2014). Relationship between self-compassion and emotional intelligence in nursing students. International Journal of Nursing Practice, 20(6), 588596. https://doi.org/10.1111/ijn.12204 
Kurebayashi, Y., \& Harada, Y. (2020). Effect of process recording and self-compassion on self-focus: A pre-post interventional pilot study. Journal of Human Sciences, 17(1), 131-141. doi:10.14687/jhs.v17i1.5753

Shattell, M. M., Hogan, B., \& Hernandez, A. R. (2006). The interpretive research group as an alternative to the interpersonal process recording. Nurse Educator, 31(4), 178-182. https://doi.org/10.1097/00006223-200607000-00014

Smeets, E., Neff, K., Alberts, H., \& Peters, M. (2014). Meeting suffering with kindness: Effects of a brief self-compassion intervention for female college students. Journal of Clinical Psychology, 70(9), 794-807. https://doi.org/10.1002/jclp.22076

Sugiyama, S., \& Asakura, K. (2017). The process of enhancing autonomous clinical judgment among Japanese nurses. Journal of Japan Academy of Nursing Science, 37(0), 141-149. https://doi.org/10.5630/jans.37.141

Sutton, A. (2016). Measuring the effects of self-awareness: Construction of the Self-Awareness Outcomes Questionnaire. Europe's Journal of Psychology, 12(4), 645-658. https://doi.org/10.5964/ejop.v12i4.1178

Svendsen, J. L., Osnes, B., Binder, P., Dundas, I., Visted, E., Nordby, H., . . Sørensen, L. (2016). Trait self-compassion reflects emotional flexibility through an association with high vagally mediated heart rate variability. Mindfulness, 7(5), 1103-1113. https://doi.org/10.1007/s12671-016-0549-1

Takano, K., \& Tanno, Y. (2008). Development of Japanese-version Rumination-Reflection Questionnaire. The Japanese Journal of Personality, 16(2), 259-261. https://doi.org/10.2132/personality.16.259

Tamura, S., Tanaka, K., Tanaka, S., Horiuchi, K. (2010). The characteristics of behavior for nursing problems according to experience year. Nihonkangogakekiironnbunnsyu: Kangokanri, 40, 246248.

Tatsumi, N., Nakama, Y., Tada, M., \& Koyama, S. (2008). The evaluation of education for nursing research as part of in-service education. Japan Journal of Nursing Science, 33, 72-76.

Townsend, M. C. (2011). Essentials of psychiatric/mental health nursing: concepts of care in evidence-based practice (5th ed.). Philadelphia: F. A. Davis Company.

Trompetter, H. R., de Kleine, E., \& Bohlmeijer, E. T. (2017). Why does positive mental health buffer against psychopathology? an exploratory study on self-compassion as a resilience mechanism and adaptive emotion regulation strategy. Cognitive Therapy and Research, 41(3), 459-468. https://doi.org/10.1007/s10608-016-9774-0

Tsuji, C., Ogasawara, C., Takeda, C., Katayama, Y., Imura, K., \& Nagayama, H. (2007). The plateau phenomena and factors related to the development of nurses practical abilities. Journal of Japan Society of Nursing Research, 30, 31-38. https://doi.org/10.15065/jisnr.20070910003 